\title{
Understanding Far Field Ultrasonic Power Transmission for Automobile Sensor Networks in Free Space
}

\author{
A. Y. S. Pandiyan ${ }^{1}$, R. La Rosa ${ }^{2}$, M. E. Kiziroglou ${ }^{1}$, E. M. Yeatman ${ }^{1}$ \\ ${ }^{I}$ Department of Electronic and Electrical Engineering, Imperial College London, London, UK. \\ ${ }^{2}$ ST Microelectronics, Catania, Italy.
}

\begin{abstract}
Ultrasonic Power Transmission (UPT) has gained attention for powering implanted diagnostic devices due to its non-invasive properties. However, UPT in free-space is still less exploited with considerable potential for powering Wireless Sensor Networks (WSNs). An important challenge in understanding the significant parameters for a UPT system is to comprehend the losses and quantify the limitations of the technology in terms of distance, frequency and transmission power (ISO226). In this work, the authors attempt to model the transmission link of an UPT system, identifying variables which can be modified for obtaining maximum power output from the wireless power transfer through formulation and experimental results. Ultrasonic transducers of two varying frequencies were used in free space power transmission to understand the absorptive and geometric attenuation of sound waves in air, experimentally. A measured power of $6.3 \mu \mathrm{W}(40 \mathrm{kHz})$ and $8.3 \mathrm{nW}(100 \mathrm{kHz})$ was observed over $30 \mathrm{~cm}$. These observations may enable acoustic powered WSNs in automobiles powering multi-nodes using single transmitter.
\end{abstract}

\section{Motivation}

Acoustic energy is already exploited in many commercial and industrial products such as ultrasonic cleaning, nondestructive testing, ultrasonic imaging and SONAR systems. However, in these applications the sound power is utilized in its purest form rather than by converting it to electrical energy. In an UTP system the transmitter transduces electrical power into pressure waves in the form of sound into a medium. A receiving transducer is positioned in the path of these pressure waves in order to convert the mechanical energy into electrical energy. The system structure of an ultrasonic power transfer system with multiple receiving node is as shown in Fig. 1.

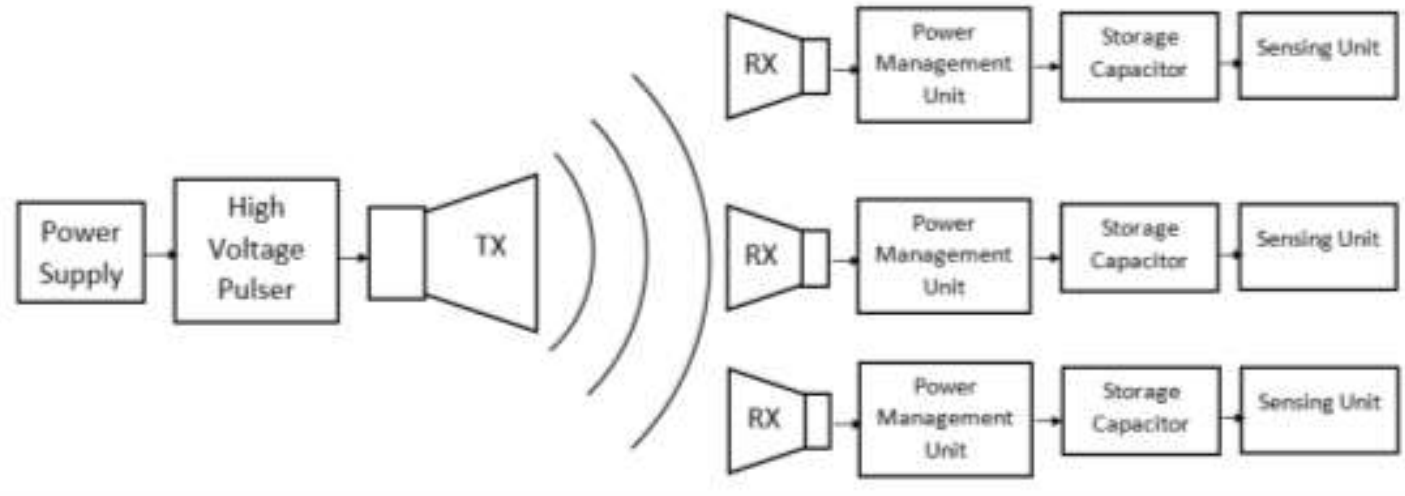

Fig. 1: Schematics of the system with a single transmitter and multi-receivers.

The main advantage of UPT when compared to other radiative power transfer is that the speed of propagation of sound is much lower and therefore have a smaller wavelength. This allows the receiver probe dimensions to be very small, taking their directionality into account. Therefore, UPT systems can be easily integrated into MEMS sensing systems allowing them to comprise rechargeable WSNs.

\section{Experimental setup and Results}

Subsequent to the proof of concept [1], this work aims to quantify the underlying design parameters to be controlled or modified with the purpose to optimize the power transmission of the wireless system. Mason proposed an equivalent piezoelectric model [2] segregating the electric and the acoustic ports through transformers, and quantifying the voltage of the transducer with respect to the force exerted by the membrane on the medium. Fig. 2 shows the equivalent circuit of the UPT link between two acoustic transducers through an Op-Amp model for the geometric and absorptive attenuation in air. The absorptive losses of ultrasonic waves in air can be modelled by the empirical formula[3],

$$
P_{d}=P_{0} e^{-\alpha d}
$$

where, $P_{d}$ is the sound power received at a distance $d$ from the transmitter sending a power $P_{0}$, and $\alpha$ is the attenuation co-efficient which depends on the material properties and frequency of the signal. 
Also, for spherical ultrasonic probes, the geometric losses can be modelled by [4],

$$
P_{d}=D \cdot \frac{P_{0} A_{R x}}{4 \pi d_{T x}^{2}}
$$

where, $D$, and $d_{T x}$ are the directivity and diameter of the transmitter respectively, and $-A_{R x}$ is the area of the receiver probe. Therefore, the cumulative losses can be obtained by the product of Eq (1) and (2).

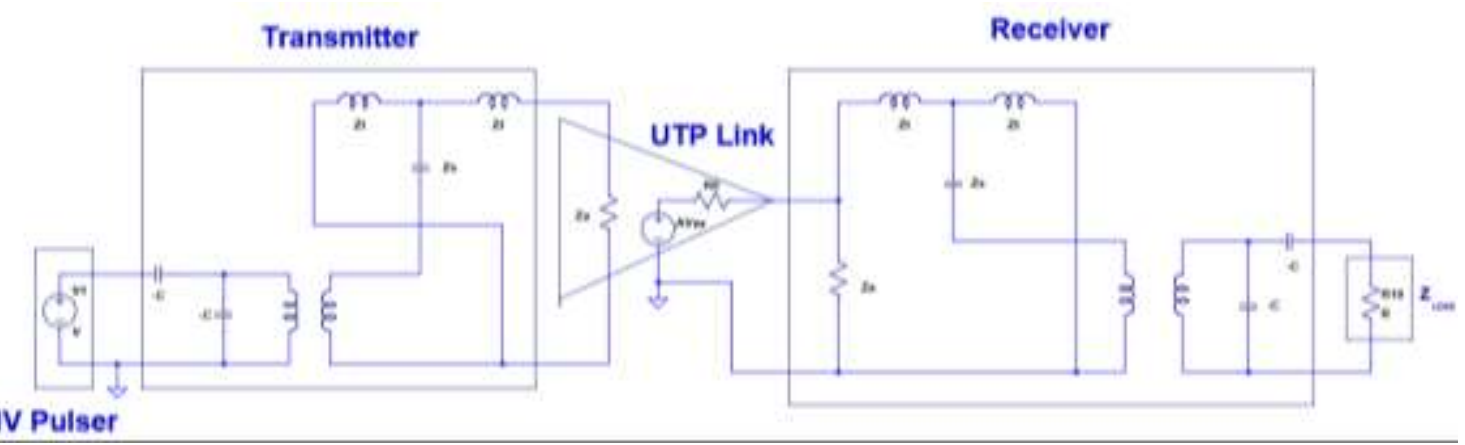

Fig. 2: Mason Model for the piezoelectric transducers (transmitter and receiver) combined by an OpAmp UPT Model.

For the experimental setup, two sets of ultrasonic sensors were used with varying frequency $(40 \mathrm{kHz}$ and $100 \mathrm{kHz}$ ) and directivity. The specifications of these transducers are summarized in Table 1.

\begin{tabular}{|c|c|c|c|}
\hline \multicolumn{4}{|c|}{ Table 1: Specifications of the Ultrasonic Transducers (UST). } \\
\hline \multicolumn{2}{|c|}{ Parameters } & UST1 & UST2 \\
\hline \multicolumn{2}{|c|}{ Model No. } & MA40S4S & MCUSD40A100B17RS-70C \\
\hline \multirow{2}{*}{ Supply Voltage } & Typ. & $20 \mathrm{~V}_{\mathrm{pp}}$ & $24 \mathrm{~V}_{\mathrm{pp}}$ \\
\hline & Max & $20 V_{p p}$ & $600 \mathrm{~V}_{\mathrm{pp}}$ \\
\hline \multicolumn{2}{|l|}{ Frequency } & $40 \mathrm{kHz}$ & $100 \mathrm{kHz} \pm 8 \mathrm{kHz}$ \\
\hline \multicolumn{2}{|l|}{ Directivity } & $80^{\circ}$ & $9^{\circ} \pm 2$ \\
\hline \multicolumn{2}{|l|}{ Capacitance } & $2550 \mathrm{pF}$ & $900 \mathrm{pF}$ \\
\hline \multicolumn{2}{|c|}{ Max. Sound Pressure Level (SPL) } & $120 \pm 3 \mathrm{~dB}$ & $130 \pm 3 \mathrm{~dB}$ \\
\hline \multicolumn{2}{|c|}{ Distance of Detection } & 0.2 to $4 \mathrm{~m}$ & 0.2 to $4 \mathrm{~m}$ \\
\hline \multicolumn{2}{|c|}{ Operating Temperature } & $-40^{\circ}$ to $80^{\circ} \mathrm{C}$ & $-20^{\circ}$ to $80^{\circ} \mathrm{C}$ \\
\hline \multicolumn{2}{|l|}{ Matching Layer } & No & Plastic \\
\hline \multicolumn{2}{|l|}{ Load Resistor } & $2.7 \mathrm{k} \Omega$ & $3 \mathrm{k} \Omega$ \\
\hline
\end{tabular}

To power the transmitter, a high-voltage ultrasonic pulser (STEVAL - IME011V2) is used to generate a pulsating square wave. The receiver and transmitter were then installed on a mount in a tripod for free space transmission. The distance between them was varied manually from $1 \mathrm{~cm}$ to $70 \mathrm{~cm}$ to characterize the power transfer behavior in near, transition and far field. The experimental setup is as shown in Fig. 3.

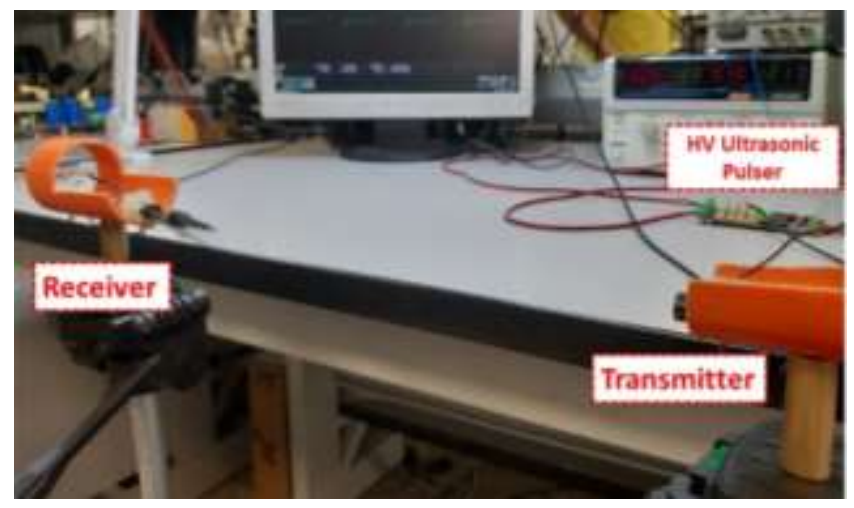

(a)

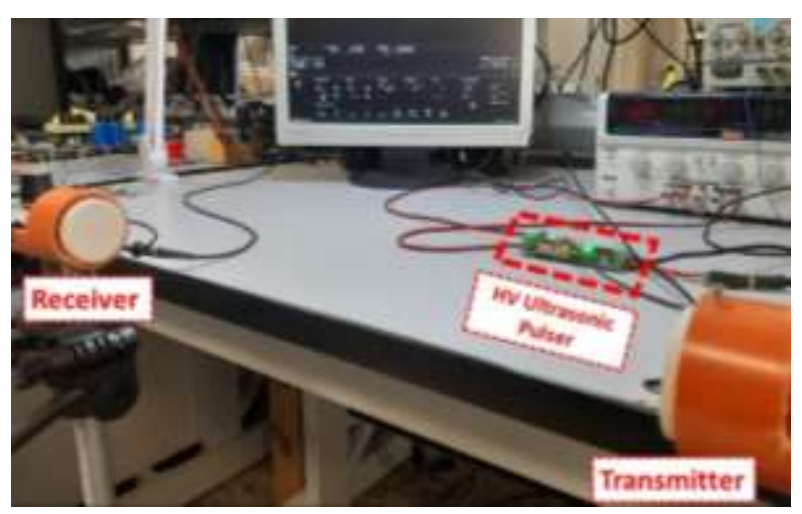

(b)

Fig. 3: Experimental Setup for the UPT system for (a) $40 \mathrm{kHz}$ transducers (b) $100 \mathrm{kHz}$ transducers. 
The voltage of the received signal from UST1 and UST2 were measured and plotted against the distance between the transmitter and the receiver in Fig. 4. It is observed that the power transmission efficiency in UST2 is inferior to UST1 by an order of an order of magnitude of 2 . This can be since the UST1 is excited at its maximum voltage, maximum sound pressure level (SPL) of $120 \mathrm{~dB}$ was reached. Therefore, the received power is higher when compared to UST2 which is operated at $1 / 25^{\text {th }}$ of its maximum SPL. However, the changes in the phase difference should be further investigated, focusing in far field region along with the pressure measurements for both the transducers.

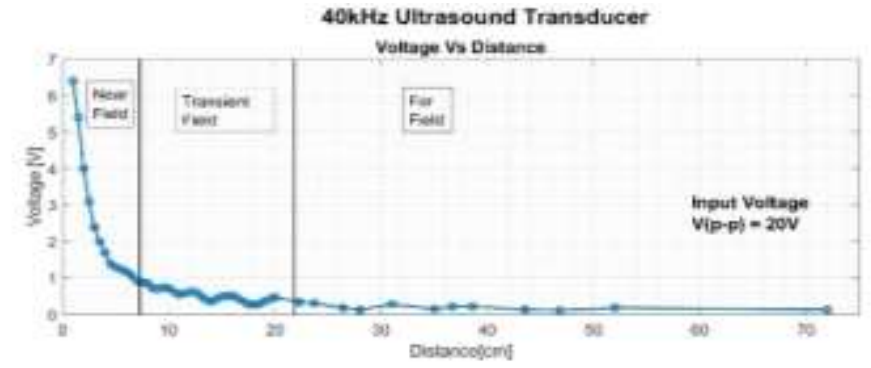

(a)

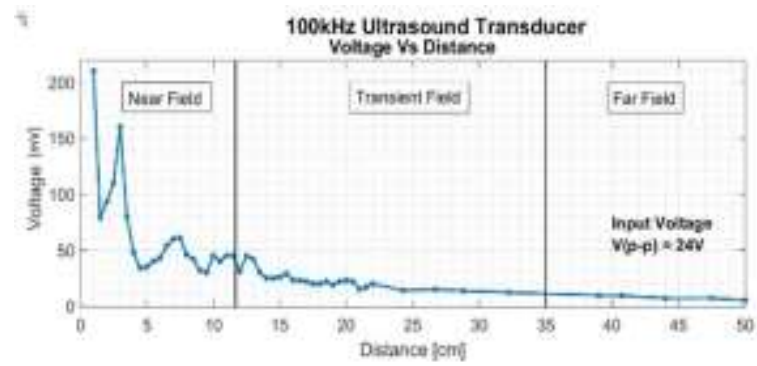

(b)

Fig. 4: Trends in the voltage and phase for (a) $40 \mathrm{kHz}$ transducers (b) $100 \mathrm{kHz}$ transducers.

The power harnessed at the receiver was also measured across a load resistor and the results obtained are plotted as a function of the separation distance as shown in Fig. 5. As can be interpreted by the performance graph of UST1, after the near field range there are prominent maxima and minima in the energy transfer. This is mainly due to the impedance mismatch caused by the reflection. This effect restricts the placement of the receiver only to certain positions for maximum energy transfer. To overcome this, some authors have implemented waveguides such as horns to reduce impedance mismatch $[5,6]$. Therefore, further research and dedicated hardware development should increase the performance of an UPT system.

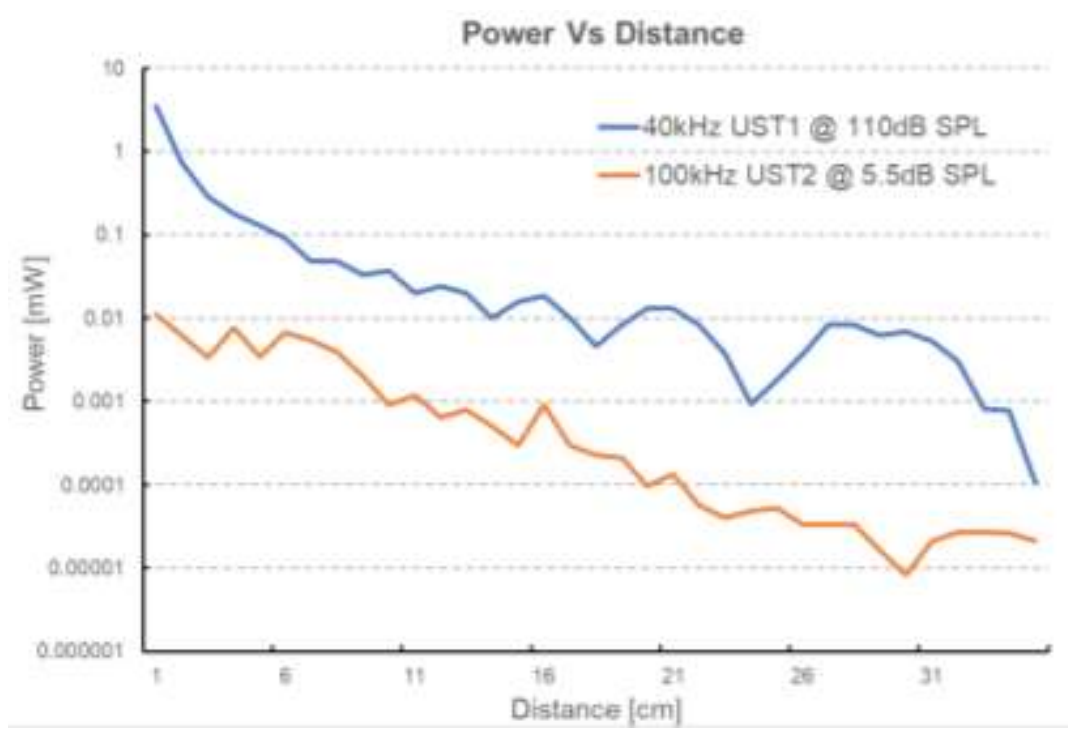

Fig. 5: Trends in the received power of UST1 and UST2 over the measured distance.

\section{Summary}

This paper demonstrates a comparison between ultrasonic power transmission at two different range of frequencies. The results were analyzed and compared for examining the possibility of automobile WSNs powering applications. Commercial $40 \mathrm{kHz}$ and $100 \mathrm{kHz}$ ultrasonic transducers were used in free space transmission and their output voltage was studied as a function of input voltage, power and distance. A power of $13 \mu \mathrm{W}$ was measured across the $2.7 \mathrm{k} \Omega$ using a $40 \mathrm{kHz}$ transducer at $20 \mathrm{~cm}$, which can be utilized for cold starting or nulling stand-by condition in WSNs. Considering this as a pioneer attempt for UPT in automobile applications, further improvements and experiments were proposed to achieve efficient power transfer.

\section{Acknowledgement}

The authors gratefully acknowledge the funding provided by the European Commission under the H2020 Marie Sklodowska Curie's ENHANCE project, Grant Agreement No: 722496 to accomplish this research. 


\section{References}

[1] M. Roes, M. Hendrix, and J. Duarte, "Contactless energy transfer through air by means of ultrasound," in IECON 2011-37th Annual Conference of the IEEE Industrial Electronics Society, 2011, pp. 1238-1243: IEEE.

[2] S. Sherrit, S. P. Leary, B. P. Dolgin, and Y. Bar-Cohen, "Comparison of the Mason and KLM equivalent circuits for piezoelectric resonators in the thickness mode," in 1999 IEEE Ultrasonics Symposium. Proceedings. International Symposium (Cat. No. 99CH37027), 1999, vol. 2, pp. 921926: IEEE.

[3] T. L. Szabo, "Time domain wave equations for lossy media obeying a frequency power law," The Journal of the Acoustical Society of America, vol. 96, no. 1, pp. 491-500, 1994.

[4] K. Nakamura, Ultrasonic transducers: Materials and design for sensors, actuators and medical applications. Elsevier, 2012.

[5] M. G. Roes, J. L. Duarte, and M. A. Hendrix, "Design of stepped exponential horns for acoustic energy transfer systems," in 2013 IEEE International Ultrasonics Symposium (IUS), 2013, pp. 306-309: IEEE.

[6] T. Ishiyama, Y. Kanai, J. Ohwaki, and M. Mino, "Impact of a wireless power transmission system using an ultrasonic air transducer for low-power mobile applications," in IEEE Symposium on Ultrasonics, 2003, 2003, vol. 2, pp. 1368-1371: IEEE.

Corresponding author: Akshayaa S. Pandiyan, 707, Electrical Engineering, South Kensington, SW7 2BT London, UK. Phone: +44 (0) 207594 6242. Mail: a.pandiyan@imperial.ac.uk 